\title{
Evaluación del Compromiso académico y Grit. Fortalezas de carácter a desarrollar en estudiantes de postgrado
}

\author{
Florencia Teresita Daura ${ }^{1}$ * \\ 1Universidad Austral, Escuela de Educación; CIIPME (CONICET); Buenos Aires, Argentina. \\ https://orcid.org/0000-0001-8101-6626 \\ María Cecilia Barni ${ }^{1}$ \\ https://orcid.org/0000-0001-9628-667X \\ Mariela Lourdes González \\ Universidad Nacional de Cuyo. Mendoza, Argentina. Mendoza, Argentina. \\ http://orcid.org/0000-0002-2649-1579 \\ Juan Alberto Assirio \\ https://orcid.org/0000-0001-8101-6626 \\ Guillermo Lúquez ${ }^{1}$ \\ https://orcid.org/0000-0002-3517-3847
}

Recibido: 06/04/2020 Revisado:04/06/2020 Aceptado: 10/06/2020

\begin{abstract}
Resumen
Este trabajo presenta los resultados de un estudio en curso que tiene como objetivo determinar la relación entre el Compromiso Académico y Grit de alumnos argentinos de postgrado. Para ello, se aplicó una metodología cuantitativa, descriptiva y correlacional, con una muestra seleccionada por conveniencia de 188 estudiantes, los cuales completaron la UWES-SS y la Escala Grit. Los resultados indican que las mujeres presentan mejores índices en las dimensiones del Compromiso Académico y en el factor Consistencia de Interés de la Escala Grit. Asimismo, se encontraron correlaciones positivas y significativas entre ambos constructos; por el contrario, no se hallaron diferencias según el postgrado que cursan los estudiantes, como tampoco entre los niveles de Compromiso académico global y el Grit global según la carrera y las horas semanales dedicadas al estudio. Estos hallazgos muestran la importancia de orientar a los alumnos con estrategias concretas.
\end{abstract}

Palabras clave: Educación del carácter, Compromiso Académico, Grit, Postgrado, Enseñanza Superior.

\section{Assessment of Academic Engagement and Grit: Strengths of Character to Be Developed in Postgraduate Students}

\footnotetext{
Abstract

This paper introduces the results of an ongoing study, aimed at describing Academic Engagement and Grit standards in students attending postgraduate studies. To comply this a quantitative, descriptive and correlational study was applied
} 
to a convenience sample of 188 students who filled out the UWES-SS and the Grit Scale. The results showed that women scored better in the Academic Engagement dimensions and the Consistence of Interest factor in the Grit Scale. In addition, significant, positive correlations were found in both constructs. Conversely, no significant differences were found among postgraduate programs, nor among global Academic Engagement and global Grit by program or weekly study time. These findings showed the relevance of guiding students with specific strategies.

Keywords: Character Education, Academic Engagement, Grit, Postgraduate Students, Higher Education

\section{Avaliação do comprometimento acadêmico e do Grit. Forças de caráter a serem desenvolvidas em estudantes de pós-graduação}

\section{Resumo}

Este trabalho apresenta os resultados de um estudo em curso que tem como objetivo determinar a relação entre o Envolvimento Acadêmico e o Grit de alunos argentinos de pós-grão. Para isso, foi aplicada uma metodologia quantitativa, descritiva de correlação, com uma mostra selecionada por conveniência de 188 alunos, os quais completaram a UWESSS e a escala Grit. Os resultados indicam que as mulheres apresentam melhores índices nas dimensões de Envolvimento Académico e no fator Consistência de Interesse da Escala Grit. Da mesma forma, foram encontradas correlações positivas e significativas entre ambas as construções. Pelo contrário, não foram achadas diferenças segundo o pós-grão que os alunos cursam, nem entre os níveis de Envolvimento Acadêmico global e Grit global segundo a carreira e as horas semanais dedicadas ao estudo. Os descobrimentos mostram a importância de orientar os alunos com estratégias concretas.

Palavras-chave: Educação do Caráter, Envolvimento Académico, Grit, Pós-Graduação, Ensino Superior.

Citar como:

Daura, F. T., Barni, M. C., Gonzales, M. L., Asirio, J. A., \& Luque, G. (2020). Evaluación del Compromiso académico y Grit. Fortalezas de carácter a desarrollar en estudiantes de postgrado. Revista Digital de Investigación en Docencia Universitaria. 14(1), e1172. https://doi.org/10.19083/ridu.2020.1172

Erase l presente trabajo aborda la vinculación entre el Compromiso Académico y el Grit, constructos que son particularmente relevantes en todos los niveles educativos y que merecen ser promovidos a través de la denominada Educación del Carácter (Bernal, González-Torres, \& Naval, 2015). En ese sentido, es necesario destacar que este trabajo se enmarca dentro del proyecto de investigación en curso titulado " $\mathrm{El}$ Compromiso Académico, la Determinación y la Orientación Temporal en estudiantes universitarios de carreras dirigidas a desarrollar el liderazgo. Su incidencia en el desempeño y en la retención académica" y recibe la financiación del Vicerrectorado de Investigación de la Universidad Austral (Argentina).

Este enfoque, que resurgió en las últimas décadas, se orienta a desarrollar en forma integral las capacidades (Almilburu, 2015) de la persona, contemplando todas sus dimensiones, tanto la cognitiva como volitivo-afectiva, la física, la social y la trascendente con el objeto de brindar una formación personal y moral a los estudiantes, con la que puedan consolidar su personalidad y su proyecto vital. Sus bases se encuentran en los aportes brindados por la Filosofía, la Psicología (especialmente de la Psicología Positiva), la Sociología, la Ética y las Neurociencias al considerarlas ciencias que ofrecen métodos y conceptos que facilitan la educación en valores y la adquisición de virtudes en los estudiantes (Naval, González-Torres \& Bernal, 2015). Por este motivo, posee un enfoque superador de modelos previos sobre la enseñanza en valores orientados a la formación ciudadana.

La Educación del Carácter se nutre de diversas 
disciplinas. Una de ellas es la Psicología Positiva, que ofrece una orientación novedosa y preventiva para explicar tanto el Compromiso Académico como el Grit. Luego de profundizarlos teóricamente, y de considerarlos a la luz de lo que ocurre en carreras universitarias de postgrado orientadas a formar competencias de liderazgo en distinto tipo de organizaciones, se plantean las siguientes interrogantes: ¿Qué diferencias existen en los niveles de Compromiso Académico y de Grit en sus estudiantes? Y ¿Cómo la universidad logra promover mayores niveles de Compromiso Académico y de Grit en sus alumnos?

Es a partir de estas preguntas que se plantean dos objetivos: describir los niveles de Compromiso Académico y de Grit que poseen los alumnos de postgrados dirigidos a desarrollar competencias de liderazgo; y proponer estrategias pedagógicas a implementar en el nivel universitario para favorecer ambas capacidades.

De acuerdo con ello, en primer lugar, se presenta un breve marco teórico sobre las variables principales de estudio, que se ubican dentro del ámbito de la Educación del Carácter. En segundo lugar, se describe el diseño metodológico adoptado, de carácter cuantitativo, que se desarrolló con una muestra no probabilística, seleccionada por conveniencia e integrada por 188 estudiantes de postgrados, que completaron un cuestionario sociodemográfico diseñado ad hoc, la Escala Ultrecth de Compromiso ocupacional para estudiantes (Utrecht Work Engagement Scale for Students, UWES-17S) (Schaufeli, Salanova, González-Romá, \& Baker, 2002), en la versión adaptada para población Argentina por Mesurado, Richaud y Mateo (2016); y la Escala Grit (Grit Scale) (Duckworth et al., 2007) que se encuentra en proceso de validación en población argentina (Tortul \& Daura, en evaluación).

Por sus características, se trata de un estudio que se destaca por su originalidad en tanto que, si bien se hallaron investigaciones centradas en el Compromiso Académico y en el Grit en población argentina, se trata de uno de los primeros trabajos formales realizados en este contexto geográfico, en el que se vinculan ambos constructos en estudiantes de postgrado y que presenta datos enriquecedores a partir de la utilización de los cuestionarios aludidos.

\section{La educación de las emociones y de las fortalezas de carácter en la universidad}

En el ámbito educativo, quienes ejercemos el rol de enseñante, aspiramos a lograr un proceso integral, dirigido a desarrollar todas las potencialidades del alumno. Algunas de estas últimas se desprenderán de cada una de las dimensiones educables del ser humano, (a) la cognitivo-intelectual, (b) la social, (c) la afectivo-motivacional, (d) la física y (e) la trascendente (Barni, 2019; Vázquez, 2012).

Desde nuestra perspectiva, este interés coincide con la presencia de dos miradas o tendencias que predominan en el desarrollo del proceso de enseñanza. La primera de ellas, que consideramos de carácter preventivo, es la que está centrada en promover todas las cualidades de cada alumno, por sus características, considera a éste como el protagonista principal del proceso, que necesita de la guía y orientación del docente. La otra, de tipo paliativo, atiende los problemas de aprendizaje -personales o no- una vez estos están instalados.

La concomitancia de ambos enfoques no es un problema de por sí. De hecho, cuando se intenta orientar a un estudiante para que resuelva alguna dificultad académica, se lo hace en pos de promover su desarrollo integral. Por el contrario, el problema se presenta cuando las energías y el tiempo se invierten más en atender este tipo de cuestiones y no en prevenirlas (Barni, 2019).

Primordialmente, la segunda mirada se inspira en el paradigma tradicional o academicista que aún hoy tiene vigencia, de acuerdo con el cual se brinda prevalencia a la transmisión y adquisición de información, conocimientos y habilidades netamente cognitivas (así, por ejemplo, en los primeros niveles se da prioridad al aprendizaje de la escritura, la lectura, el cálculo; y en los siguientes a los contenidos esperados a cada etapa y/o carrera) y al desarrollo de una de las dimensiones educables del alumno (la intelectual), menoscabando las demás que propician el autoconocimiento, el desarrollo de fortalezas o hábitos personales, la proyección de un proyecto de vida (como es la dimensión afectivo-motivacional); salir de sí mismo y trascenderse a fin de realizar aportes en el contexto sociocultural del que se forma parte, y de brindarse a otros (Barni, 2019). 
La prevalencia de este enfoque ha hecho que el proceso educativo se centre solo en la obtención de números objetivos que den cuenta de un cierto nivel de éxito, descuidando el desarrollo del bienestar del alumno, del claustro docente y del sistema familiar. Este planteo no conlleva el menosprecio de una de las tareas fundamentales de la institución educativa, que se concreta en la transmisión de contenidos conceptuales, esenciales para el crecimiento personal y para la inserción del hombre en el mundo social, laboral y profesional, sino que nos permite remarcar que ésta debe ir de la mano de otras acciones con tendencia a propiciar el autoconocimiento y el desarrollo de fortalezas de carácter a enseñar, perfeccionar y evaluar, a fin de que cada estudiante consolide un proyecto vital (Barni, 2019; Roegiers, 2010).

Un giro al respecto, lo ofrece el enfoque de la Psicología Positiva aplicada a la educación. Efectivamente, en la actualidad, son numerosos los trabajos y esfuerzos realizados para aplicar, en el ámbito de la enseñanza formal e informal, varios de los constructos abordados por esta disciplina, que posee tan solo 20 años de antigüedad (Palomera Martín, 2017).

Algunos de estos constructos se estudian en torno a los 3 pilares básicos que sostienen esta disciplina (Seligman, 2002): (a) las emociones positivas, (b) los rasgos positivos (entre los que se encuentran las virtudes, las fortalezas personales y las habilidades) y (c) las instituciones u organizaciones educativas, que favorecen o no el desarrollo de estas emociones y rasgos.

Podemos decir que el desarrollo de las emociones y de los rasgos positivos de carácter son la llave personal para alcanzar mayores niveles de satisfacción vital en todos los ámbitos en los que se desenvuelve el sujeto. Claro está que este proceso no se da en solitario, sino en interacción con otros e insertos en un contexto institucional determinado. De hecho, el tercer pilar mencionado, la institución de enseñanza, constituye el lugar en el cual la persona, desde sus primeros años de vida hasta la universidad, pasa la mayor parte del tiempo fuera del ámbito familiar.

Al respecto, Palomera Martín (2017), explica que aquellas escuelas que brindan más actividades extracurriculares, tareas significativas según la edad de los alumnos y un entorno seguro, predi- cen mayores niveles de felicidad en los niños. Esta afirmación nos ayuda a comprender cómo en los últimos años se efectuaron muchísimos esfuerzos para introducir en la enseñanza formal contenidos vinculados con la inteligencia emocional, con el desarrollo de emociones, de hábitos o virtudes y de fortalezas del carácter (Arguis Rey, Bolsas Valero, Hernández Paniello, \& Salvador Monje, 2012; Mayer, Salovey, Caruso, \& Cherkasskjy, 2015; Pascual Ferris \& Cuadrado Bonilla, 2001; Renom Plana, 2003; Seligman, Erns, Gilham, Reivich, \& Kinkins, 2009; Soldevila, 2009).

Pero ¿Qué ocurre en las instituciones de nivel superior, particularmente en el de postgrado? ¿Cuáles son las fortalezas de carácter que, en última instancia, repercuten en la obtención de mayores niveles de rendimiento o de permanencia académica? ¿Qué niveles en cada una de ellas presentan los alumnos de postgrado? Estos interrogantes nos permiten considerar cómo entre las fortalezas del carácter existen particularmente dos que guardan una vinculación directa con la obtención de un mejor rendimiento y con la continuidad académica por parte de los estudiantes y que serán profundizadas en los siguientes apartados: el Compromiso Académico y el Grit.

\section{El Compromiso Académico}

El Compromiso Académico es un metaconstructo que ha sido abordado desde distintas líneas teóricas (Daura, 2015) y que guarda una directa vinculación con el abordaje de la deserción y la retención. Esto le permite la oportunidad de estudiar el fenómeno complejo de la permanencia estudiantil desde un enfoque positivo y preventivo. En ese sentido, así como los dos primeros constructos se centran en estudiar los factores que inciden en el abandono o en la continuidad en los estudios, el compromiso o el involucramiento, se focaliza en analizar los aspectos personales, actitudinales o contextuales que favorecen a que el estudiante se sienta parte del proceso de aprendizaje en el que participa, de la institución de la que forma parte y que la organización educativa realice acciones que colaboren a ello (Tinto, 2006-2007).

Si bien su estudio comenzó a realizarse en la década de los 80 a partir de los trabajos de Astin (1984), sobre la participación del estudiantado, existen numerosas definiciones sobre el mismo. 
En este contexto tomamos como referencia la que brinda Schaufeli (Schaufeli, Martínez, Marqués-Pinto, Salanova, \& Bakker, 2002; Schaufeli, Salanova, et al., 2002) en línea con los planteos de la Psicología Positiva.

Este autor la comprende "como un estado mental positivo relacionado con el trabajo y con la actividad académica, que se caracteriza por el vigor, la dedicación y la absorción" (Schaufeli, Salanova, et al., 2002, p. 79), variables que se manifiestan a través de una energía, resistencia y esfuerzo, independientemente de las dificultades que se presenten; el entusiasmo y la inspiración por estudiar; y una gran capacidad de concentración para realizar la tarea.

En Latinoamérica y, en forma particular en la República Argentina, la mayoría de los trabajos efectuados sobre el Compromiso Académico se realizaron con alumnos de los primeros niveles del sistema educativo (Arguedas Negrini, 2010, 2011; Dabenigno, Larripa, Austral, Tissera, \& Goldenstein, 2010; Dabenigno, Larripa, \& Austral, 2012; Dabenigno \& Tissera, 2011; Larripa, 2012; Rigo, 2013, 2016, 2019; Rigo \& Donolo, 2014a; Rigo \& Donolo, 2014b). En los últimos años también comenzó a estudiarse en el nivel superior (Daura, 2015; 2016; Daura, Baravalle, \& Barni, 2016; Daura \& Durand, 2018; Mesurado et al., 2015; Mesurado, Tortul, \& Schonfeld, 2018; Rigo, 2019), aspecto de crucial importancia ya que permite profundizar su vinculación con la permanencia y con el rendimiento académico, como con otras variables psicopedagógicas que influyen en el proceso de aprendizaje. Entre estas últimas se presenta la oportunidad de analizar la vinculación que guarda con el Grit, constructo de reciente abordaje en el contexto geográfico mencionado.

\section{Grit: pasión y perseverancia para alcanzar metas a largo plazo}

El Grit, que posee diversas traducciones (valor, determinación, coraje, firmeza, aguante, entre otras), fue acuñado por Duckworth (2016) con el objetivo de hacer referencia a la pasión y la perseverancia para alcanzar metas a largo plazo. En coincidencia con estudios previos (Barni \& Daura, 2019; Daura, Barni, González, Assirio, \& Lúquez, 2019), en el presente trabajo, optamos por mencionarlo de la misma forma en que lo denomina su autora original, es decir, como sustantivo propio.

$\mathrm{Su}$ abordaje cuenta tan solo con una trayectoria de 12 años, reflejada en la existencia de numerosos trabajos de reciente publicación (Dusworth, Peterson Matthews, \& Kelly, 2007; Ducworth \& Quinn, 2009; Robertson-Kraft \& Dusckorth, 2014) y que muestran el interés que existe por éste, al analizar el peso que ejerce en la obtención de un buen rendimiento en diversos aspectos del desarrollo personal, el deportivo, el profesional y, en especial, el educativo (Christopoulou, Lakioti, Pezirkianidis, Karakasidou, \& Stalikas, 2018; Duckworth, Kirby, Tsukayama, Berstein, \& Ericsson, 2010; Duckworth, Quinn, \& Seligman, 2009; Eskreis-Winkler, Shulman, \& Duckworth, 2014; Perkins-Gough, 2013; Yeager, Henderson, Paunesku, Walton, D'Mello, Spitzer, \& Duckworth, 2014).

Su desarrollo es independiente del nivel de cociente intelectual que se posee, ya que a través de diversos estudios se corroboró que las personas que se destacan en su profesión no lo hacen tanto por sus cualidades innatas, sino por el esfuerzo y la dedicación que emplean para alcanzar objetivos (Duckworth et al., 2007).

De su definición se desprenden las dos variables que lo conforman: (a) consistencia de interés y (b) perseverancia en el esfuerzo que hacen referencia, respectivamente, a la tendencia a mantener los objetivos que se hayan planteado, y a trabajar intensamente ante los contratiempos y dificultades.

Se encuentran puntos de contacto entre el Compromiso Académico y el Grit si se considera que el motivo fundamental por el cual el primero surgió, fue comprender y encontrar estrategias para prevenir la deserción estudiantil, y promover la perseverancia necesaria para culminar los estudios (Office of Educational Technology, 2013).

Al respecto, son varios los trabajos en los que se muestra la relación existente entre la determinación y el Compromiso Académico (Atapattu, 2015; Hodge, Wright \& Bennett, 2018; entre otros).

El propósito principal del estudio fue determinar la relación entre el Compromiso Académico y el Grit de estudiantes de distintos postgrados. Además, se plantean como objetivos específicos: a) determinar si existen distintos niveles de Compromiso Académico y de Grit en función del sexo de los estudiantes que participaron en el estudio. 
b) Corroborar si existen correlaciones positivas y significativas entre los niveles de compromiso y de Grit que poseen los estudiantes, según el sexo. c) Indagar si los estudiantes poseen distintos niveles de Compromiso Académico y de Grit en función del postgrado que se encuentran cursando; y d) corroborar si presentan vinculaciones significativas entre los niveles globales de Compromiso Académico y de Grit en función de la carrera que se encuentran cursando y las horas de estudio dedicadas durante la semana.

\section{Método}

\section{Diseño}

El trabajo adoptó un enfoque cuantitativo, con el objeto de analizar la realidad estudiada con datos numéricos. En este contexto, se optó por utilizar un diseño de carácter descriptivo y correlacional, con la finalidad de conocer y describir la relación existente entre las variables de estudio (Hernández Sampieri, Fernández Collado, \& Baptista Lucio, 2010).

\section{Participantes}

Se optó por realizar el estudio con población universitaria de postgrados, al considerar dos aspectos fundamentales, por un lado, las posibilidades de acceso que se tenía a la misma; y por el otro, la necesidad de cubrir una laguna de conocimiento, al no existir estudios previos en estudiantes argentinos de esa franja etaria en los que se indague la vinculación entre los dos constructos principales de estudio.

A partir de ello, se conformó una muestra no probabilística y por conveniencia, integrada por 188 estudiantes, de acuerdo a los permisos concedidos por sus instituciones de pertenencia. Los sujetos que integraron la muestra cursaban carreras de postgrado orientadas a la formación de competencias de liderazgo; entre ellos 120 (63,8\%) eran mujeres y $68(36,2 \%)$ varones, y su edad promedio era de 40,63 años. De los grupos mencionados, 123 pertenecían a un postgrado (P1) dirigido a formar directivos de instituciones educativas que poseían una titulación previa de nivel terciario, con un plan de estudios de 2 años de duración. 34 eran estudiantes de un postgrado (P2) de 2 años de duración, que también se encuentra orientado a formar directivos del mismo tipo de organizaciones, pero que poseían una titulación previa de grado universitario. Es importante aclarar que los alumnos de estas dos carreras, en su mayoría, ocupaban un rol directivo en sus ámbitos laborales en simultaneidad con la cursada del postgrado. Otros 6 estudiantes cursaban una maestría en dirección de empresas (P3), con modalidad intensiva, con lo que la cursaban sin desempeñarse laboralmente en ninguna organización. Estas tres carreras de postgrado son impartidas por una institución de gestión privada, que se ubica en la provincia de Buenos Aires (Argentina)

Por su parte, 25 alumnos cursaban un postgrado (P4) orientado a formar directivos de instituciones militares, con un plan de estudios de 1 año de duración, que tenían una titulación universitaria de base. Este postgrado es impartido por la Universidad de Defensa Nacional, que es la responsable de formar a todo el cuerpo de oficiales de las Fuerzas Armadas Argentinas; por sus características, es de gestión estatal. Cabe destacar que este postgrado incide de manera cierta en el desarrollo profesional de los estudiantes, por lo que culminarlo implica la obtención de mejores puestos de trabajo a futuro. Si bien la muestra de estudiantes resulta muy heterogénea, tiene en común el hecho de encontrarse cursando carreras orientadas a formar competencias de liderazgo para ejercerlas en organizaciones de diverso tipo. Para análisis posteriores, a fines prácticos, a esta muestra se la reorganizó en tres submuestras conformada por 26 estudiantes del P1 y 27 del P2, que se seleccionaron en forma aleatoria, y los 25 alumnos del P4. De estos estudiantes, 34 eran mujeres $(43,4 \%)$ y 44 varones $(56,4 \%)$, y su edad promedio era de 40,96 años.

\section{Técnicas de recolección de datos Cuestionario sociodemográfico}

Se diseñó un cuestionario con el fin de recoger información referente a diversas variables sociodemográficas. Particularmente, se pidió información referente a: 1 . la edad; 2 . el sexo; 3 . el estado civil; 4 . la institución de pertenencia; 5 . la carrera de postgrado cursada; 6. el año de ingreso; el beneficio económico recibido por parte de la univer- 
sidad; 7. el nivel educativo al que se aspira alcanzar; 8. la ocupación actual y; 9. las horas semanales dedicadas al estudio. De todas estas variables, en el presente trabajo se consideraron cuatro, en concreto: la 2., la 4., la 5., y la 9.

\section{Escala Utrecht sobre compromiso para estudiantes}

Se aplicó la Escala Utrecht sobre compromiso hacia el trabajo para estudiantes, en su adaptación para estudiantes de la Utrecht-Work Engagement Scale (UWES-SS) (Schaufeli, Martínez et al., 2002; Schaufeli, Sanlanova et al., 2002), adaptada y validada en población argentina (Mesurado et al., 2016) arrojando coeficientes Alfa elevados para cada una de las dimensiones del instrumento y para la variable de Compromiso académico global: Vigor: 0,76; Dedicación: 0,89; Absorción: 0,74; Compromiso académico global: 0,88. De la misma manera, el Análisis Factorial Confirmatorio, mostró la existencia de los tres factores propuestos por los autores originales (Schaufeli, Martínez et al., 2002) y los que realizaron la validación en Argentina.

El instrumento posee un diseño de escala tipo Likert, con 7 opciones de respuesta que van de 0 (nunca) a 6 (siempre) y está conformado por 14 ítems que evalúan tres dimensiones del Compromiso Académico: Vigor (5 ítems), que se refiere a la presencia de altos niveles de energía y resistencia mental durante el desarrollo de una tarea, como así también a la disposición a esforzarse y persistir en ella incluso ante dificultades; Dedicación (5 ítems) que remite al sentido de importancia, entusiasmo, inspiración, orgullo y reto que la tarea le proporciona; y Absorción (4 ítems), que se refiere al estado de concentración y de felicidad que se experimenta mientras se realiza la tarea. Un alto nivel de Compromiso Académico se corresponde con altas puntuaciones en las tres dimensiones. Para utilizarlo en análisis posteriores, se calculó el Compromiso Académico global, fruto del cual, los sujetos que obtienen una puntuación inferior a 3 puntos son considerados con bajo nivel de involucramiento.

\section{Escala Grit-Original}

La escala Grit-Original (Grit-O), diseñada por Ducworth et al. (2007), en su adaptación para pobla- ción argentina (Tortul \& Daura, en prensa), en la cual, el análisis de consistencia interna del instrumento arrojó un Alfa de 0,83 para la totalidad de la Escala; 0,80 para el factor Consistencia de intereses (CI) y 0,79 para el factor Perseverancia de interés (PI); asimismo, los 12 ítems que la integran mostraron poseer una adecuada capacidad discriminativa al obtener diferencias altamente significativas ( $p=0.000$ ); a su vez, el Análisis Factorial Exploratorio, confirmó la existencia de los dos factores propuestos por los autores (Duckworth et al., 2007), explicando el 41,98\% de la variancia. El primer factor explicó el 31,53\% de la variancia y el segundo explicó el 10,45\%.

El instrumento posee un diseño de escala tipo Likert, de 5 opciones y está conformada por 12 ítems, distribuidos en forma equitativa en dos factores: Consistencia de interés (CI): que explora la tendencia a mantener los objetivos e intereses que se hayan propuesto. Perseverancia de esfuer$z o(P E)$ : que mide la inclinación a trabajar intensamente ante los contratiempos y las dificultades. El instrumento permite obtener un nivel global de Grit, que oscila entre 1 y 5 puntos, que corresponden, respectivamente, a sujetos que poseen un nivel muy bajo y alto en la capacidad. Para el propósito del presente estudio, consideramos que los individuos poseen una puntuación baja si alcanzan una valoración inferior a 2,50 puntos, por ser éste el puntaje medio entre el mínimo y el máximo que arroja la escala.

\section{Procedimientos}

Los instrumentos fueron completados por los estudiantes en situaciones regulares de clase, ante la presencia de los investigadores, en días y horarios acordados con las autoridades de las instituciones educativas. Los estudiantes que integraron la muestra participaron en forma voluntaria. Para ello, en primer lugar, se les informaron los objetivos de la investigación, se les garantizó la confidencialidad de los datos recogidos y se les solicitó la firma de un consentimiento informado.

\section{Análisis de datos}

Por la naturaleza del estudio, se calculó el valor promedio de las dimensiones que integran el UWES-SS y la Escala Grit. Del mismo modo, se calculó el puntaje del Compromiso académico global 
y del Grit global al sumar los valores de todos los ítems que los conforman y dividiéndolos por el número total de indicadores.

Para realizar los análisis estadísticos se conformó una base en Excel que luego se importó al paquete estadístico IBM SPSS versión 23.0. A continuación, y respetando el orden de los objetivos específicos planteados, se efectuaron sucesivos análisis de acuerdo al siguiente detalle: a) a fin de analizar las diferencias existentes entre los niveles de Compromiso Académico y de Grit en función del sexo de los estudiantes que conformaron la muestra, se realizaron análisis descriptivos y la prueba $t$ de Student para muestras independientes. b) Para confirmar si existen o no correlaciones positivas y significativas entre los niveles de Compromiso Académico y de Grit que poseen los estudiantes, según su sexo, se efectuaron análisis descriptivos y una correlación de Pearson. c) Para explorar si se presentan diferencias significativas entre los niveles de Compromiso Académico y de Grit que poseen los alumnos en función del postgrado que cursan, se emplearon las submuestran seleccionadas de la muestra total a fin de contar con submuestras comparables entre sí, y se efectuaron análisis de varianza (ANOVA, Análysis of Variance) de un factor. d) A fin de corroborar si se presentan o no vinculaciones significativas entre los niveles de Compromiso Académico Global y de Grit Global en función de la carrera y las horas de estudio dedicadas por los alumnos durante la semana, se realizó un análisis multivariante de varianza (MANOVA, Multivariate Analysis of Variance). A fin de obtener el tamaño del efecto y la potencia estadística de algunos de los resultados alcanzados, se utilizó el paquete estadístico G*Power.

\section{Resultados}

\section{Niveles de Compromiso Académico y de Grit: análisis descriptivo y diferencias según el sexo} Los resultados (Tabla 1) indican que las mujeres que participaron en el estudio poseen índices más elevados tanto en las subescalas que conforman la UWES-SS y los factores de la Escala Grit. En lo que respecta al primer cuestionario, se destacan por tener una mayor dedicación $(M=5,11)$ frente al es-

Tabla 1.

Descriptivos de las dimensiones del UWES-SS y de los factores de la escala Grit, según el sexo

\begin{tabular}{|c|c|c|c|c|c|c|c|}
\hline Variables & Dimensiones & Sexo & $\mathbf{N}$ & Min & Máx & $M$ & $D E$ \\
\hline \multirow{8}{*}{$\begin{array}{l}\text { Compromiso } \\
\text { Académico }\end{array}$} & \multirow{2}{*}{ Vigor } & Mujeres & 120 & 1,60 & 6,00 & 4,48 & 0,915 \\
\hline & & Hombres & 68 & 0,60 & 6,00 & 4,05 & 1,18 \\
\hline & \multirow{2}{*}{ Dedicación } & Mujeres & 120 & 2,00 & 6,00 & 5,29 & 0,76 \\
\hline & & Hombres & 68 & 0,60 & 6,00 & 4,80 & 1,05 \\
\hline & \multirow{2}{*}{ Absorción } & Mujeres & 120 & 1,50 & 6,00 & 4,63 & 1,00 \\
\hline & & Hombres & 68 & 0,50 & 6,00 & 4,06 & 1,23 \\
\hline & \multirow{2}{*}{ CA } & Mujeres & 120 & 1,97 & 6,00 & 4,81 & 0,77 \\
\hline & & Hombres & 68 & 1,17 & 6,00 & 4,30 & 1,03 \\
\hline \multirow{6}{*}{ Grit } & \multirow{2}{*}{$\mathrm{PE}$} & Mujeres & 120 & 2,33 & 5,00 & 4,15 & 0,47 \\
\hline & & Hombres & 68 & 2,33 & 5,00 & 4,13 & 0,54 \\
\hline & \multirow{2}{*}{ CI } & Mujeres & 120 & 1,83 & 5,00 & 3,64 & 0,63 \\
\hline & & Hombres & 68 & 1,33 & 4,67 & 3,42 & 0,65 \\
\hline & \multirow{2}{*}{ Grit } & Mujeres & 120 & 2,08 & 4,83 & 3,89 & 0,42 \\
\hline & & Hombres & 68 & 1,83 & 4,83 & 3,78 & 0,52 \\
\hline
\end{tabular}

Nota. CA: Compromiso académico, PE: Perseverancia de esfuerzo, CI: Consistencia de interés 
tudio y a toda situación de aprendizaje, manifestando entusiasmo e inspiración, al considerarlas un desafío en sí mismo.

En lo que se refiere a los factores que integran la Escala Grit, poseen una mayor perseverancia de esfuerzo $(M=4,15)$, lo que indica que se aplican con constancia para alcanzar las metas que se proponen, con independencia de los obstáculos o de las dificultades que se presenten.

Estos resultados se corroboran con el análisis t de Student para muestras independientes, que arrojó diferencias estadísticamente significativas a favor de las mujeres en las tres dimensiones de la UWES-SS: en Vigor ( $t=2,76 ; p=0,006$, IC95\%[0.12; 0.73]), 1- $\beta=.75, d=.40$; en Dedicación ( $t=3,70: p=$ $.000,1$ IC95\%[0.23; 0.75]), 1- $\beta=.94, d=.53$; en Absorción $(t=3,43 ; \mathrm{p}=.0001$, IC95\%[0.24; 0.89]), $1-\beta=$
.94, d= .54; y en el Compromiso Académico global $(t=3,768 ; p=.0001$, IC95\%[0.23; 0.76]), 1- $\beta=.91$, $\mathrm{d}=.50$. Por su parte, en la Escala Grit, sólo alcanzaron una diferencia significativa a su favor en el factor Consistencia de interés $(t=2,207 ; p=.0029$, IC95\%[0.22; 0.40]), $1-\beta=.58, d=.33$.

\section{Correlación entre las dimensiones del Compromiso Académico y los factores del Grit según el sexo}

A fin de analizar la correlación existente entre los niveles de Compromiso Académico y de Grit que poseen los estudiantes, según su sexo, se calcularon los índices de correlación $r$ de Pearson, significancia estadística, tamaño del efecto $(p)$ y potencia estadística (1- $\beta$ ) (ver Tabla 2). En todos los casos se encontraron correlaciones significativas

Tabla 2

Correlaciones entre las dimensiones de la UWES-SS y los factores de la Escala Grit según el sexo

\begin{tabular}{|c|c|c|c|c|c|c|c|}
\hline Dimensiones & 1 & 2 & 3 & 4 & 5 & 6 & 7 \\
\hline Vigor & &, $667^{* *}$ &, $808^{* *}$ & ,912** &, $424^{* *}$ & $0,328^{* *}$ & $0,427^{* *}$ \\
\hline$p$ & & 0.81 & 0.89 & 0.95 & 0.65 & 0.57 & 0.65 \\
\hline $1-\beta$ & & 1 & 1 & 1 & 1 & 0.99 & 1 \\
\hline Dedicación &, $588^{* *}$ & & ,686** & ,847** & ,406** & $0,269^{*}$ & ,381** \\
\hline$p$ & 0.76 & & 0.82 & 0.92 & 0.63 & 0.51 & 0.61 \\
\hline $1-\beta$ & 1 & & 1 & 1 & 1 & 0.99 & 1 \\
\hline Absorción &, $622^{* *}$ &, $711^{* *}$ & & ,931** &, $436^{* *}$ & $0,269^{*}$ & $0,397^{* *}$ \\
\hline$p$ & 0.78 & 0.84 & & 0.96 & 0.66 & 0.51 & 0.63 \\
\hline $1-\beta$ & 1 & 1 & & 1 & 1 & 0.99 & 1 \\
\hline $\mathrm{CA}$ & $858^{* *}$ & $866^{* *}$ & ,890** & & ,469** & $0,323^{* *}$ & ,448** \\
\hline$p$ & 0.92 & 0.93 & 0.94 & & 0.68 & 0.56 & 0.66 \\
\hline 1- $\beta$ & 1 & 1 & 1 & & 1 & 0.99 & 1 \\
\hline $\mathrm{PE}$ &, $253^{* *}$ &, $311^{* *}$ & ,255** &, $314^{* *}$ & &, $510^{* *}$ &, $842^{* *}$ \\
\hline$p$ & 0.50 & 0.55 & 0.50 & 0.56 & & 0.71 & 0.91 \\
\hline $1-\beta$ & 1 & 0.99 & 0.99 & 0.99 & & 1 & 1 \\
\hline CI &, $251^{* *}$ & $0,184^{*}$ & ,215* &, $251^{* *}$ & 0,125 & & ,893** \\
\hline$p$ & 0.50 & 0.42 & 0.46 & 0.59 & & & \\
\hline 1- $\beta$ & 0.99 & 0.99 & 0.99 & 0.99 & & & \\
\hline Grit & ,333** &, $315^{* *}$ & ,307** &, $367^{* *}$ & ,657** & ,830** & \\
\hline$p$ & 0.57 & 0.56 & 0.55 & 0.69 & 0.81 & 0.91 & \\
\hline $1-\beta$ & 0.99 & 0.99 & 0.99 & 0.99 & 1 & 1 & \\
\hline
\end{tabular}

Nota: en la diagonal superior se detallan las correlaciones obtenidas en la muestra de los varones; y en la inferior, en la muestra de las mujeres. ${ }^{* *} p \leq .01 .{ }^{*} p \leq .05$. 
y positivas, tanto en las mujeres como en los varones entre las variables que conforman la UWES-SS entre sí, entre los factores de la Escala Grit y entre las variables. La excepción se presenta en las alumnas, entre los dos factores de la escala Grit que no tienen ninguna vinculación significativa. En cambio, al efectuar el mismo análisis con la muestra total, la correlación positiva sí se presenta; en efecto, la Perseverancia de esfuerzo $r$ (188) $=0,279^{* *}, p=0,01$ se asocia positivamente con la Consistencia de Interés.

Estos resultados parecen indicar que a mayor energía para realizar una tarea y disposición frente al esfuerzo (Vigor), ante más entusiasmo para realizar una tarea (Dedicación), y concentración y alegría mientras se la ejecuta (Absorción), los sujetos son propensos a mantener el interés (Consistencia de interés) frente a las metas previamente fijadas y a trabajar con intensidad para lograrlas (Perseverancia de esfuerzo), independiente de las dificultades que se presenten.

\section{Comparación entre las dimensiones de la UWES- SS, los factores de la Escala Grit y el postgrado de los estudiantes}

Se efectuó un análisis de varianza (ANOVA one way) a fin de comprobar si existen diferencias en- tre las dimensiones de la UWES-SS y los factores de la Escala Grit en función al postgrado que los estudiantes estuvieran cursando (ver Figura 1). Para efectuar este análisis, se tomaron como variables dependientes las variables que conforman cada instrumento y, como variable independiente, las submuestras de carreras que se seleccionaron de la muestra general que participó en el estudio; de esta forma, se logró obtener 3 grupos homogéneos a los cuales poder comparar con mayor precisión (P1; P 2 y P 4).

El valor para el modelo, para la dimensión Vigor fue de $[F(2,75) 0.123, p>.88, f=0,57]$; para Dedicación de $[\mathrm{F}(2,75) 2.183, \mathrm{p}>.120, f=0,90]$; para Absorción [F $(2,75)$ 0.862, p >.426, $f=0.74]$; para Compromiso académico global $[F(2,75) 872, p$ >.422, $f=0,74]$; para Perseverancia de esfuerzo de [F $(2,75) 713, \mathrm{p}>.493, f=0,72]$; para Consistencia de interés de $[F(2,75) 1.664, \mathrm{p}>.196, f=0,85]$; y para Grit global de $[F(2,75) 1.455, \mathrm{p}>.240, f=0,83]$. Se efectuó Scheffe como análisis Post hoc, sin encontrarse diferencias significativas entre los grupos.

No obstante, se produce un efecto cruzado entre los alumnos del P1 y del P4 en las subescalas del UWESS-S y los factores del Grit. En concreto, los estudiantes del P1 se ven más favorecidos en los índices del Compromiso Académico, mientras

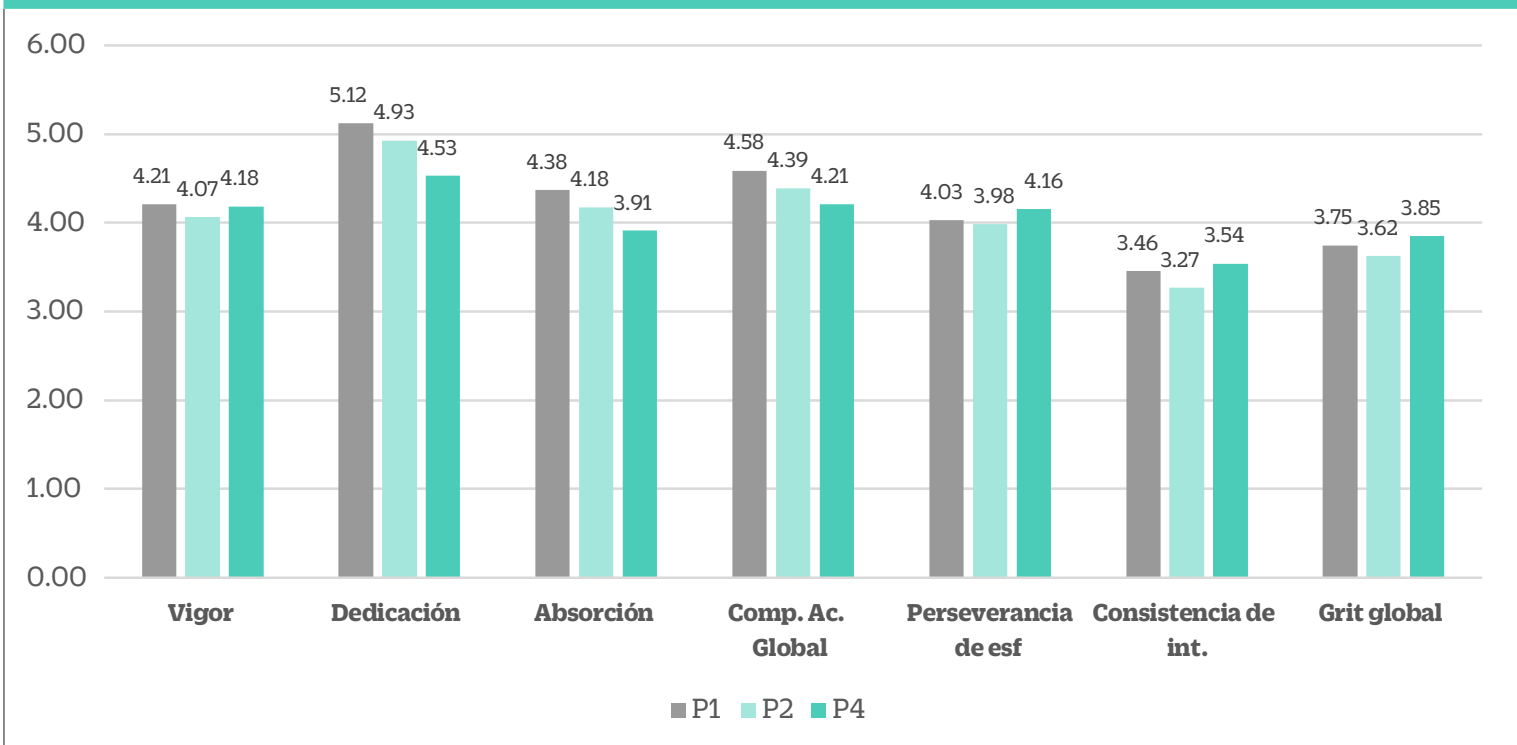

Figura 1. Comparación entre las dimensiones de la UWES-SS, los factores de la Escala Grit y el postgrado. Nota: elaboración propia 
que los del P4 tienen mayores ventajas para perseverar y mantener el interés por alcanzar las metas académicas.

\section{Comparación entre el Compromiso Académico global y el Grit global en función del postgrado y de las horas de estudio dedicadas durante la semana}

Se llevó a cabo un análisis MANOVA para confirmar si existen diferencias estadísticamente significativas entre el Compromiso Académico global y el Grit global en función del postgrado cursado por los estudiantes y las horas de estudio dedicadas durante la semana. A tal fin, como variables dependientes se tomaron las dos primeras dimensiones mencionadas, y como variables independientes las carreras de postgrado y las horas de estudio dedicadas durante la semana.

Para facilitar el análisis de los datos, a estas últimas se las categorizó en tres niveles: 1 . De 0 a 5 horas; 2. De 6 a 10 horas; y 3. A partir de 11 horas para estudiar de lunes a viernes.

En este caso, si bien tampoco se encontraron diferencias estadísticamente significativas, en los 3 postgrados se aprecia que a mayor carga horaria semanal dedicada al estudio, mayor es el nivel de Compromiso Académico y de Grit global alcanzado por los alumnos (figura 2).

\section{Discusión}

El estudio del Compromiso Académico y del Grit, y particularmente, su vinculación, ocupa un lugar preponderante en el ámbito académico y educativo; al ser competencias o fortalezas de carácter a ser promovidas en el marco de la Educación del Carácter.

A través de las líneas desarrolladas se refuerza la importancia de promover un proceso educativo integral, que contemple el desarrollo de todas las potencialidades del alumno, haciendo especial hincapié en todas las dimensiones del ser humano (cognitiva, social, afectivo-motivacional, física y trascendente), y no centrándose solo en una o alguna de ellas. De esta manera, sin menoscabar la transmisión de conocimientos, es crucial que en la enseñanza formal también se promueva la dimensión social y afectiva de los educandos, a través del

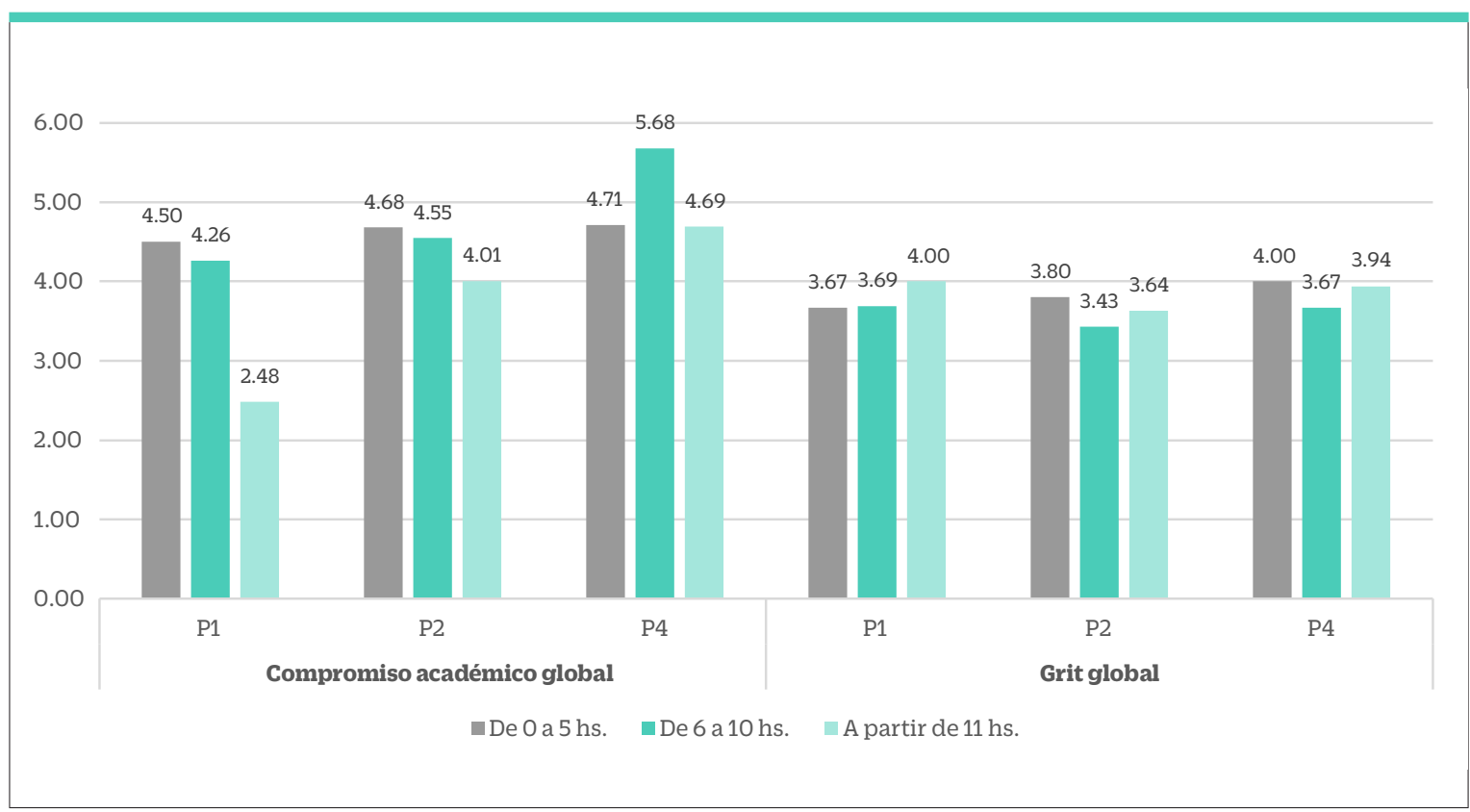

Figura 2. Comparación entre el Compromiso académico global y el Grit global, según del postgrado y horas de estudio durante la semana. Nota: elaboración propia 
desarrollo de las emociones y de los rasgos positivos de carácter tanto en los primeros niveles del sistema educativo, como en el superior. Este último, hoy más que nunca, debe priorizar la promoción de una mayor autonomía en sus estudiantes, que se refleje no solo en sus resultados académicos, sino también en otros aspectos de su desempeño personal, que son aquellos que en definitiva tendrán que ponerse en juego en el ámbito laboral y social.

Es precisamente en este ámbito en el que se desarrolló el presente trabajo, haciendo foco, en particular, en el estudio del Compromiso Académico y del Grit, al ser competencias o fortalezas de carácter que favorecen la permanencia y el rendimiento académico, y que es factible que presenten distintas características en los estudiantes de acuerdo con su sexo y con la carrera que se encuentren cursando.

En línea con estas ideas, los resultados obtenidos a través de la aplicación de la Escala Utrecht sobre compromiso para estudiantes (UWES-SS) y la Escala Grit, dirigidos a evaluar, en un caso, tres dimensiones del Compromiso Académico, y en el otro, dos factores del Grit o de la tenacidad, muestran que existen diferencias de acuerdo con el sexo de los estudiantes de postgrado.

En este sentido, las mujeres son las que alcanzaron mejores resultados en las tres dimensiones del UWES-SS y en el factor Consistencia de Interés de la Escala Grit, efectos que coinciden con los obtenidos en otros estudios, en los que las alumnas se destacan por poseer mayores puntuaciones en la Escala Grit (Bazelais, Lemay, \& Doleck, 2016; Chaustre Jota, 2018; Christensen \& Knezek, 2014; Rojas, Reser, Usher, \& Toland, 2012) y que estarían vinculados con la predisposición natural del sexo femenino hacia la autodisciplina, el esfuerzo, la tenacidad y la obtención de mayores niveles de rendimiento académico.

En coincidencia con estudios previos (Hodge et al., 2018) se comprobó que existen correlaciones positivas y significativas entre las subescalas de la UWES-SS y los factores de la Escala Grit, lo que permite explicar que, a mayores niveles de involucramiento académico, mayores niveles de Grit, y viceversa. La única excepción que se presentó, en las mujeres, fue entre los factores que integran la Escala Grit, que no presentan una correlación positiva y significativa. Este efecto podría estar vin- culado con la estructura interna del instrumento, tal como lo señalan Cerda, Saiz y Vergara (2018).

En forma simultánea, para interpretar con mayor precisión estos resultados, se consideran las sugerencias de Cohen (1988), de acuerdo con el cual, para interpretar las correlaciones, se considera que los valores que se encuentran entre los 0,50 y 1,00 puntos presentan una correlación fuerte; los que se encuentran entre los 0,30 y los 0,50 puntos, una correlación moderada; los que se ubican entre los 0,10 y los 0,30 puntos, una correlación débil; y los que tienen entre 0,00 y 1,00 puntos, una correlación nula.

De esta manera, los valores encontrados en ambos sexos, entre la dimensión Vigor y Dedicación, Absorción y Compromiso Académico global presentan una correlación positiva y significativa fuerte; presentándose el mismo efecto entre los factores de la Escala Grit y entre estos y el Grit global. En cambio, en las mujeres y en los varones, se encontraron correlaciones moderadas y débiles, entre las dimensiones de la UWES-SS y los factores de la Escala Grit. Con lo cual, en el caso de considerar estrategias que favorezcan el desarrollo de una u otra fortaleza en los alumnos, ello beneficiará el incremento de la otra habilidad. Estos efectos también permiten vincular las variables propias del involucramiento del modelo de Shaufeli (Schaufeli, Salanova et al., 2002) con las variables que conforman el Grit. En este sentido, tanto el vigor, como la dedicación y la absorción, serían formas a través de las cuales se manifiesta la Perseverancia y la Consistencia de Interés, permitiendo así que un sujeto logre alcanzar metas a largo plazo, que conllevan, por la distancia en el tiempo, regular el esfuerzo, perseverar, tener fortaleza y tenacidad.

Si se observan con detenimiento los resultados del Compromiso Académico y del Grit en función del postgrado que cursan los estudiantes, la ausencia de diferencias estadísticamente significativas podría estar vinculada a la etapa del proceso vital en la que se encuentran, la adultez. Desde esa perspectiva, la motivación para participar en un proceso educativo formal o informal, está asociada a la satisfacción de metas vitales y al enriquecimiento interior (como ser promovido, adaptarse al medio laboral, resolver inconvenientes del mundo del trabajo; adquirir más conocimientos; poder 
servir mejor a otros) (D’anna \& Hernández, 1998), lo que coincidiría con índices elevados de compromiso y de Grit. A la par de ello, estos efectos estarían relacionados con las características de los alumnos que realizan cada uno de los postgrados y que, en el caso de los del P1 y del P2, trabajan al tiempo que asisten a clases; mientras que, los del P4, tienen una dedicación exclusiva al estudio.

Una inferencia similar podría asociarse tras corroborar que no se presentan diferencias significativas entre el Compromiso académico global y el Grit global según la carrera de los alumnos y las horas que dedican durante la semana al estudio. No obstante, se destaca el hecho que la inversión de más horas para el estudio está vinculada a mayores niveles de Compromiso y de Grit, efecto que concuerda con investigaciones previas (Duckworth et al., 2007). Del mismo modo, las horas que se invierten para estudiar durante la semana podrían tener alguna asociación con el estilo de enseñanza que se utiliza en cada postgrado, tópico que ha sido abordado en otras indagaciones (Barni, 2019) y que podría ahondarse en futuros trabajos para profundizar el estudio del Compromiso Académico y del Grit.

\section{Conclusiones}

Los resultados recabados, por un lado, permitieron alcanzar los objetivos planteados. En este sentido, se encontraron diferencias significativas en las variables estudiadas según el sexo y se hallaron correlaciones entre ellas. Sin embargo, no se encontraron diferencias estadísticamente significativas entre los niveles de Compromiso Académico y de Grit en los alumnos de las submuestras conformadas para el caso; del mismo modo, tampoco se encontraron vinculaciones significativas entre los niveles de Compromiso Académico Global y de Grit Global en función de la carrera y las horas dedicadas al estudio.

De esta manera, los hallazgos abren el camino para considerar algunas estrategias a implementar en las instituciones educativas para favorecer en los alumnos de postgrado el desarrollo de ambas fortalezas de carácter.

La primera de ellas se orienta a brindar forma- ción teórica y práctica sobre los constructos principales de estudio del presente trabajo a los docentes y tutores, a fin de que puedan estimularlas en los alumnos en los espacios de aprendizaje que tienen a cargo.

La segunda de ellas se dirige a efectuar un seguimiento de las capacidades aludidas a través de la aplicación de los instrumentos UWES-SS y de la Escala Grit, en sus versiones adaptadas para población argentina, en estudiantes de grado y de postgrado, con el objeto de utilizar los datos recogidos para efectuar mejoras en el proceso de aprendizaje que se desarrolla en las aulas o fuera de ellas. El desarrollo de esta estrategia conllevará la participación colaborativa de distintas áreas de la universidad, como el área de evaluación de la calidad, la de orientación pedagógica y la de bienestar estudiantil, entre otras.

La tercera estrategia se podría aplicar en las entrevistas individuales y/o grupales del espacio de tutoría. En concreto, se refiere a focalizarlas a trabajar aspectos derivados de las competencias de Compromiso Académico y Grit. Con respecto a las primeras, podrá abordarse la capacidad para regular el esfuerzo frente a las dificultades que se presentan en la vida académica y laboral; el entusiasmo y la satisfacción para realizar las actividades solicitadas en el postgrado. Las segundas podrán enfocarse en el tratamiento de temáticas referidas a la capacidad para seleccionar metas académicas, personales y profesionales superadoras, capaces de ser alcanzadas. Asimismo, sería conveniente complementar las temáticas tratadas en las entrevistas con otras actividades que se organicen en la agenda universitaria y que permitan integrarlas a la vida familiar, profesional y social de los alumnos.

La cuarta estrategia se refiere a la aplicación en cada materia, y en su diseño pedagógico-didáctico, de las sugerencias dadas en el punto anterior. De esta manera, alcanzar una meta lejana en el tiempo, como por ejemplo rendir, cursar la asignatura y aprobar el examen final, es probable que se logre con más facilidad a partir del planteo de objetivos a corto plazo (Moritz, 2015²) -haciendo uso de la estrategia denominada SMART, en la que

1 Moritz (2015) denomina a esta estrategia SMART y distingue en ella diversos aspectos. 
se distinguen diversos aspectos- que involucren a todo el grupo de estudiantes, así se promueve en forma simultánea el aprendizaje colaborativo, la autorregulación y se evita la procrastinación.

Por la naturaleza propia del trabajo desarrollado y por la muestra con la que se lo efectuó, los resultados alcanzados, si bien no son generalizables a la población objeto de estudio, son relevantes de por sí, al obtener una descripción sobre el perfil de los alumnos participantes y sentar las bases para investigaciones futuras. Entre estas, caben mencionar: los efectos que tienen ambos constructos en el rendimiento, en la permanencia académica y en competencias de liderazgo; estudios cuasiexperimentales en los que se analicen los resultados de una experiencia pedagógica orientada a promover el Compromiso Académico y el Grit. En paralelo, también se recomienda promover indagaciones sobre estas temáticas en las que se conformen muestras más representativas de la población objeto de estudio, incluyendo estudiantes de diversas instituciones, que pertenezcan a otros contextos sociales y geográficos del país, que cursen otras carreras y formen parte de otros niveles del sistema educativo.

Más allá de las limitaciones encontradas, en sí mismo, el presente trabajo constituye un aporte para fomentar el desarrollo del Compromiso Académico y del Grit en los estudiantes de postgrado de argentina. El objetivo: diseñar y aplicar estrategias que favorezcan su involucramiento con el aprendizaje y el desarrollo de capacidades que son necesarias en el medio laboral y social.

\section{Referencias}

Amilburu, M. G. (2015) ¿Competencias, capacidades o virtudes? Matices importantes en el lenguaje educativo. Pedagogía e Vita, 73, 37-52.

Arguedas Negrini, I. (2010). Involucramiento de las estudiantes y los estudiantes en el proceso educativo. Revista Iberoamericana sobre Calidad, Eficacia y Cambio en Educación, 8(1), 63-78. https://doi. org/10.35362/rie5621534

Arguedas Negrini, I. (2011). Recursos docentes para favorecer el involucramiento de estudiantes de educación secundaria en el proceso educativo. Revista
Iberoamericana de Educación, 56 (2), 1-12. https:// doi.org/10.35362/rie5621534

Arguis Rey, R., Bolsas Valero, A. P., Hernández Paniello, S., \& Salvador Monje, M. (2012). Programa Aulas Felices. Psicología Positiva aplicada a la Educación. Equipo SATI. Recuperado de https://www.educacion.navarra.es/documents/27590/203401/Aulas+felices+documentaci\%C3\%B3n.pdf/3980650d-c22a-48f8-89fc095acd1faa1b

Astin, A. W. (1984). Student Involvement: A Developmental Theory for Higher Education. Journal of College Student Development, 25, 297-308.

Atapattu, R. (2015). Grit and Engagement as Mediators of the Relation between Academic Support and Literacy Achievement in Ethnic Minority Elementary School Students. Master of Arts. University of Maryland. Recuperado de https://goo.gl/KZQBSG

Barni, M. C. (2019) Estilos Educativos y Actitud de Vida en Adultez Temprana y Media. Universidad Católica Argentina. No publicado.

Barni, M. C. \& Daura, F. T. (2019). Is Grit the Key Element to Improve the Life Attitude? A Study with Military Students from Argentina. European Journal of Social Sciences, 2(3), 30-41. https://doi.org/10.26417/ ejss.v2i3.p30-41

Bazelais, P., Lemay, D.J., \& Doleck, T. (2016). How does grit impact college students' academic achievement in science? European Journal of Science and Mathematics Education, 4(1), 33-43.

Bernal, A., González-Torres, M.C., \& Naval, C. (2015). La Educación del carácter. Perspectivas internacionales. Participación Educativa, (segunda época), 4(6), 35 45.

Cerda, C., Saiz, J.L., \& Vergara, D. (2018). Tenacidad en estudiantes universitarios chilenos: Un estudio inicial de su estructura y red nomológica. Interdisciplinaria, 35(2), 409-423. https://doi.org/10.16888/ interd.2018.35.2.10

Christensen, R. \& Knezek, G. (2014). Comparative Measures of Grit, Tenacity and Perseverance, International Journal of Learning, Teaching and Educational Research, 8(1), 16-30.

Christopoulou, M., Lakioti, A., Pezirkianidis, C., Karakasidou, E., \& Stalikas, A. (2018). The Role of Grit in Education: A Systematic Review. Psychology, 9, 2951-2971. https://doi.org/10.4236/psych.2018.915171

Cohen, J. (1988). Statistical power analysis for the behavioral sciences. Hillsdale, N.J: L. Erlbaum Associates.

D’anna, S. \& Hernández, L. (1998). Introducción a la Psi- 
copedagogía Laboral. Círculos de Aprendizaje. Buenos Aires: Ed. Aprendizaje Hoy.

Dabenigno, V. \& Tissera, S. (2011). La escuela es un buen lugar. El involucramiento social y afectivo de los estudiantes, Boletín de la Educación Porteña, 11, agosto, 1-8. Recuperado de: https://goo.gl/DRGhZv

Dabenigno, V., Larripa, S., \& Austral, R. (2012). Inclusión educativa e involucramiento escolar. Perspectivas y prácticas en cuatro escuelas estatales de la Ciudad de Buenos Aires. Argentina en el escenario latinoamericano actual: debates desde las ciencias sociales, VII Jornadas de Sociología de la Universidad Nacional de La Plata. Facultad de Humanidades y Ciencias de la Educación. La Plata. Recuperado de: https://goo.gl/jQfnsg

Dabenigno, V., Larripa, S., Austral, R., Tissera, S., \& Goldenstein Jalif, Y. (2010). Permanencia e involucramiento escolar de los estudiantes secundarios. Perspectivas y acciones en cuatro escuelas estatales de la Ciudad de Buenos Aires. Ciudad Autónoma de Buenos Aires: Dirección Operativa de Investigación y Estadística, Ministerio de Educación. Recuperado de: https://www.buenosaires.gob.ar/areas/educacion/dirinv/pdf/permanenciainvolucramientosecundarios2010.pdf

Daura, F. \& Durand, J. (2018). ¿Cuán involucrado estoy? Un estudio exploratorio sobre el compromiso académico y la orientación temporal en estudiantes argentinos. Revista Panamericana de Educación, 20(1), 73-95.

Daura, F. T. (2015). El estudio del compromiso académico. Panorama general sobre su abordaje. Diálogos Pedagógicos, XIII (25), 54-75.

Daura, F. T. (2016). El compromiso emocional de los estudiantes hacia el contexto educativo. Su vinculación con factores temporales y con variables demográficas. Revista de Orientación Educacional, 30(58), 5473.

Daura, F. T., Barni, Ma. C., González, M., Assirio, J., \& Lúquez, G. (2019). Temples de acero, comprometidas. Una aproximación al estudio del compromiso académico y del Grit en estudiantes de postgrados orientados al desarrollo del liderazgo. Oros, L. XVII Reunión Nacional y VI encuentro internacional de la Asociación Argentina de Ciencias del Comportamiento, Posadas (Misiones).

Daura, F., Baravalle, C., \& Barni, M. C. (2016). ¿Cómo influyen la perspectiva temporal y los estilos de personalidad en el compromiso académico? Resultados de una experiencia piloto en estudiantes de carreras orientadas a la formación docente y directiva, $\mathrm{Du}$ rand, J. et al., Aprender a Enseñar: el Desafío de la Formación Docente Inicial y Continua. VII Jornadas Académicas de la Escuela de Educación de la Universidad Austral. Pilar (Buenos Aires), Recuperado de: http://cort.as/-SOiF

Duckworth, A. (2016). Grit: el poder de la pasión y de la perseverancia. Barcelona: Ediciones Urano.

Duckworth, A. \& Quinn, P. (2009). Development and validation of the Short Grit Scale (GRIT-S). Journal of personality assessment, 91(2), 166-174. https://doi. org/10.1080/00223890802634290

Duckworth, A., Kirby, T., Tsukayama, E., Berstein, H., \& Ericsson, K. A. (2010). Deliberate practice spells success: Why grittier competitors triumph at the National Spelling Bee. Social Psychological and Personality Science, 2(2), 174-181. https://doi/ 10.1177/1948550610385872

Duckworth, A., Peterson, C., Matthews, M., \& Kelly, D. (2007). Grit: Perseverance and Passion for Long-Term Goals. Journal of Personality and Social Psychology, 92(6), 1087-1101. https://doi.org/10.1037/00223514.92.6.1087

Duckworth, A., Quinn, P., \& Seligman, M. (2009). Positive predictors of teacher effectiveness. The Journal of Positive Psychology: Dedicated to furthering research and promoting good practice, 4(6), 540-547. https://doi.org/10.1080/17439760903157232

Eskreis-Winkler, L., Shulman, E., Beal, S., \& Duckworth, A. (2014). The grit effect: Predicting retention in the military, the workplace, school and marriage. Frontiers in Psychology, 5(36), 1-12. https://doi. org/10.3389/fpsyg.2014.00036

Hernández Sampieri, R., Fernández Collado, C., \& Baptista Lucio, P. (2014). Metodología de la investigación. México: McGraw Hill.

Hodge, B., Wright, B., \& Bennet, P. (2018). The Role of Grit in Determining Engagement and Academic Outcomes for University Students. Research in Higher Education, 59(4), 448-460. https://doi.org/10.1007/ s11162-017-9474-y

Larripa, S. (2012). Trabajo e involucramiento docente en escuelas secundarias con proyectos de inclusión, Boletín de la Educación Porteña № ${ }^{\circ}$, Ministerio de Educación del GCBA.

Mayer, J. D., Salovey, P., Caruso, D. R., \& Cherkasskiy, L. (2015). "La inteligencia emocional", en P. Fernández-Brocal; N. Extremera; R. Palomera; D. Ruiz-Aranda; J. M. Sal- 
guero; R. Cabello (Coord). De la neurona a la felicidad. Diez propuestas desde la inteligencia emocional. Santander: Fundación Botín, pp. 9-32.

Mesurado, B., Tortul, C., \& Schonfeld, F. (2018). Cinco grandes rasgos de personalidad: su relación con el flujo y el compromiso académico. Contextos de educación, 20(25), 150-158.

Mesurado, M. B., Richaud, M. C., \& Mateo, N. (2016). Engagement, Flow, Self-Efficacy, and Eustress of University Students: A Cross-National Comparison Between the Philippines and Argentina. The Journal of Psychology, 150(3), 281-299. https://doi.org/10.1080/00 223980.2015.1024595

Moritz, A. (2015). Building Students Skills that lead to resilience and academic tenacity. Center for Schools and Communities. Recuperado de https://tinyurl. com/rtejztm

Naval, C., González Torres, Ma.C. \& Bernal, A. (2015). Character Education. International Perspectives. Pedagogia e Vita, 73, 155-184.

Office of Educational Technology (2013). Promoting grit, tenacity, and perseverance: critical factors for success in the 21st Century. Office of Educational Technology. U.S. Department of Education. Recuperado de: https://tinyurl.com/oay8abz

Palomera Martín, R. (2017). Psicología Positiva en la escuela. Un cambio con raíces profundas. Papeles del Psicólogo, 38(1), 66-71. https://doi.org/10.23923/pap. psicol2017.2823

Pascual Ferris, V., \& Cuadrado Bonilla, M. (2001). Educación emocional. Programa de actividades para educación secundaria obligatoria. Barcelona: Praxis.

Perkins-Gough, D. (2013). The Significance of Grit: A Conversation with Angela Lee Duckworth. Educational Leadership, 71(1), 14-20.

Renom Plana, A. (Coord.) (2003). Educación emocional. Programa para la educación primaria (6 a 12 años). Barcelona: Praxis.

Rigo, D. (2013). Compromiso hacia las tareas académicas: diseños instructivos e inteligencias múltiples. Ikastorratza, 10, julio, 1-15. Recuperado de https://goo. gl/DQvBEB

Rigo, D. (2016). Desafiando las Estructuras. En Busca de un Mayor Compromiso. Anuario de investigaciones de la Facultad de Psicología. II Congreso Internacional de Psicología, V Congreso Nacional de Psicología “Ciencia y profesión”, vol. 3, núm. 1, 33-34. Recuperado de https://tinyurl.com/wlcrc4z

Rigo, D. (2019). Los entornos educativos: impacto sobre el compromiso de niños y jóvenes. Revista Educación y Ciencia, 8(51), 64-71. Recuperado de https://tinyurl.com/vdxhkgt

Rigo, D. \& Donolo, D. (2014a). Entre pupitres y pizarrones. Retos en educación primaria: el aprendizaje con compromiso. Educatio Siglo XXI, 32(2), pp 59-80. https:// doi.org/10.6018/j/202161

Rigo, D. \& Donolo, D. (2014b, noviembre). Factores clave en la promoción del compromiso en educación primaria. Un estudio sobre tasks, teachers and students. Asenjo, J.; Macías, O. \& Toscano, J. Congreso Iberoamericano de Ciencia, Tecnología, Innovación y Educación. Buenos Aires, Argentina. Recuperado de https://tinyurl.com/rh9k8ve

Robertson-Kraft, C., \& Duckworth, A. (2014). True Grit Trait-level Perseverance and Passion for Long-term Goals Predicts Effectiveness and Retention among Novice Teachers. Teachers College Record, 116(3), 1-27.

Roegiers, X. (2010). Una pedagogía de la integración. Competencias e integración de los conocimientos en la enseñanza. (Trad. Utrilla, J. J.) (2da ed., $3^{a}$. tirada). México: Educación y Pedagogía.

Rojas, J., Reser, Ma. J., Usher, E., \& Toland, M. (2012). Psychometric properties of the academic grit scale. Lexington: University of Kentucky.

Schaufeli, W., Martínez, I., Marques-Pinto, A., Salanova, M., \& Bakker, A. (2002). Burnout and engagement in university students: A Cross-National Study. Journal of Cross-Cultural Studies, 33(5), 464-481. https:// doi.org/10.1177/0022022102033005003

Schaufeli, W., Salanova, M., González-Romá, V., \& Bakker, A (2002). The measurement of burnout and engagement: A confirmatory factor analytic approach. Journal of Happiness Studies, 3, 71-92. https://doi. org/10.1023/A:1015630930326

Seligman, M., Ernst, R., Gillham, J., Reivich, K., \& Linkins, M. (2009). Positive education: positive psychology and classroom interventions. Oxford Review of Education, 35(3), 203-311. https://doi. org/10.1080/03054980902934563

Seligman. M. (2002). La auténtica felicidad. Barcelona: Ediciones B.

Soldevila, A. (2009). Emociónate. Programa de educación emocional. Madrid: Pirámide.

Tinto, V. (2006-2007). Research and practice of student retention: What next? Journal of College Student Retention, 8(1), 1-19. https://doi.org/10.2190/4YNU4TMB-22DJ-AN4W 
Tortul, M. C. \& Daura, F. (en evaluación). Validación argentina de la escala Grit. Revista de Orientación y Psicopedagogía.

Vázquez, S. M. (2012). La filosofía de la educación. Estado de la cuestión y líneas actuales. Buenos Aires: CIAFIC Ediciones.
Yeager, D., Henderson, M., D'Mello, S., Paunesku, D., Walton, G., Spitzer, B., \& Duckworth, A. (2014). Boring but important: a self-transcendent purpose for learning fosters academic self-regulation. Journal of personality and social psychology, 107(4), 559-80. https://doi.org/10.1037/a0037637

RIDU / Revista Digital de Investigación en Docencia Universitaria / e-ISSN: 2223-2516

(c) Los autores. Este artículo es publicado por la Revista Digital de Investigación en Docencia Universitaria del Área de Institutional Research and Effectiveness de la Dirección de Aseguramiento de la Calidad, Universidad Peruana de Ciencias Aplicadas. Este es un artículo de acceso abierto, distribuido bajo los términos de la LicenciaCreativeCommons Atribución-CompartirIgual 4.0 Internacional.( http://creativecommons.org/licenses/by-sa/4.o/), que permite el uso no comercial, distribución y reproducción en cualquier medio, siempre que la obra original sea debidamente citada. 